May 31, 1993

\title{
Toward a Unified Magnetic Phase Diagram of the Cuprate Superconductors
}

\author{
Alexander Sokol ${ }^{(1-3)}$ and David Pines ${ }^{(1)}$ \\ ${ }^{(1)}$ Department of Physics and ${ }^{(2)}$ Materials Research Laboratory, \\ University of Illinois at Urbana-Champaign, Urbana, IL 61801-3080 \\ (3) L.D. Landau Institute for Theoretical Physics, Moscow, Russia
}

\begin{abstract}
We propose a unified magnetic phase diagram of cuprate superconductors. A new feature of this phase diagram is a broad intermediate doping region of quantumcritical, $z=1$, behavior, characterized by temperature independent $T_{1} T / T_{2 \mathrm{G}}$ and linear $T_{1} T$, where the spin waves are not completely absorbed by the electron-hole continuum. The spin gap in the moderately doped materials is related to the suppression of the low-energy spectral weight in the quantum disordered, $z=1$, regime. The crossover to the $z=2$ regime, where $T_{1} T / T_{2 \mathrm{G}}^{2} \simeq$ const, occurs only in the fully doped materials.
\end{abstract}

PACS: 74.65.+n, 75.10.Jm, 75.40.Gb, 75.50.Ee

Typeset Using REVTEX 


\section{Introduction}

Recent measurements [1,2,3,4] of the spin-echo decay rate, $1 / T_{2 \mathrm{G}}$, for a number of cuprate oxides, taken together with earlier measurements of spin-lattice relaxation rate $1 / T_{1}$, provide considerable insight into their low frequency spin dynamics. In this communication we show how these measurements may be combined with straightforward scaling arguments to obtain a unified magnetic phase diagram for the Y- and La-based systems.

In the presence of strong antiferromagnetic correlations at a wavevector $\mathbf{Q}$, the main contribution to both $T_{1}^{-1}$ and $T_{2 \mathrm{G}}^{-1}$ for copper comes from small $\widetilde{\mathbf{q}}=\mathbf{q}-\mathbf{Q}$, so that one may write [5]:

$$
\frac{1}{T_{1} T} \sim \int d^{2} \widetilde{\mathbf{q}} \lim _{\omega \rightarrow 0} \frac{\chi^{\prime \prime}(\widetilde{\mathbf{q}}, \omega)}{\omega}, \quad \frac{1}{T_{2 \mathrm{G}}} \sim\left[\int d^{2} \widetilde{\mathbf{q}} \chi^{2}(\widetilde{\mathbf{q}}, 0)\right]^{1 / 2},
$$

where $\chi(\widetilde{\mathbf{q}}, \omega)$ is the electronic spin susceptibility near $\mathbf{Q}$. On making use of quite straightforward scaling arguments, when applicable, one may substitute $\chi(\widetilde{\mathbf{q}}, \omega)=\xi^{2-\eta} \hat{\chi}(\widetilde{\mathbf{q}} \xi, \omega / \bar{\omega})$ into Eq.(四) $(-\eta$ is the scaling dimension of the real space spin correlator) and obtain:

$$
\frac{1}{T_{1}} \sim T \xi^{-\eta} \bar{\omega}^{-1}, \quad \frac{1}{T_{2 \mathrm{G}}} \sim \xi^{1-\eta}, \quad \frac{T_{1} T}{T_{2 \mathrm{G}}} \sim \xi \bar{\omega} .
$$

where $\xi$ is the correlation length and $\bar{\omega}$ an appropriate energy scale.

\section{Applicable Scaling Regimes}

We consider first a clean quantum antiferromagnetic insulator (referred to as "insulator" hereafter), described by the $S=1 / 2$ Heisenberg model with the exchange coupling $J$. Because the spin stiffness, $\rho_{s} \simeq 0.18 J$ [6], is small compared to $J$, the quantum-critical (QC) scaling regime [7], where the only energy scale is set by temperature, $\bar{\omega} \sim T$, exists over a substantial temperature range $2 \rho_{s} \lesssim T \lesssim J$ [8,9]. The dynamical exponent, $z$, which relates the characteristic energy and length scales according to $\bar{\omega} \sim \xi^{-z}$, is $z=1$ as a consequence of Lorentz invariance at $T=0$, reflected in the linear dispersion relation of the spin waves. In this case, $T_{1} T / T_{2 \mathrm{G}} \sim \bar{\omega} \xi \sim \xi^{1-z} \simeq$ const. Since $\xi \sim \bar{\omega}^{-1 / z} \sim T^{-1 / z} \sim T^{-1}$, one further obtains 
$1 / T_{1} \sim T \xi^{z-\eta} \sim T^{\eta}$ [9] and $1 / T_{2 \mathrm{G}} \sim \xi^{1-\eta} \sim T^{\eta-1}$; because the critical exponent $\eta$ is negligible, $1 / T_{1} \simeq$ const, while $1 / T_{2 \mathrm{G}} \sim T^{-1}$.

A second regime of interest is the two-dimensional renormalized classical (RC) regime, $T_{N} \lesssim T \lesssim 2 \rho_{s}$, which is characterized by an exponential increase of the correlation length and relaxation rates. In the dynamical scaling theory of Chakravarty, Halperin, and Nelson [7], $1 / T_{1} \sim T^{3 / 2} \exp \left(2 \pi \rho_{s} / T\right)$ and $1 / T_{2 \mathrm{G}} \sim T \exp \left(2 \pi \rho_{s} / T\right)$. The prefactors arising from the $\log \xi$ corrections lead to a power-law temperature dependence of the ratio $T_{1} T / T_{2 \mathrm{G}} \sim T^{1 / 2}$, while $z=1$ leads to the cancellation of the leading (exponential) terms.

According to numerical calculations for the insulator in the 2D $S=1 / 2$ Heisenberg model [10,11], as long as $T \lesssim J$, the damping, $\gamma_{q}$, of the high energy $\left(\omega_{q} \gtrsim c \xi^{-1}\right)$ spin wave excitations, is small throughout the Brillouin zone; hence, for both $\mathrm{RC}$ and $\mathrm{QC}$ regimes those can be treated as good eigenstates of the model. The dynamical susceptibility can then be well approximated as:

$$
\chi(\mathbf{q}, \omega)=\phi_{q}\left(\frac{1}{\omega-\omega_{q}+i \gamma_{q}}-\frac{1}{\omega+\omega_{q}+i \gamma_{q}}\right),
$$

except near the origin, where the dynamics is diffusive as a consequence of total spin conservation, and near the Neel ordering vector, $\mathbf{Q}=(\pi / a, \pi / a)$, where it is relaxational; for $\xi^{-1} \lesssim \widetilde{q} \lesssim a^{-1}$, the expression (3) is valid in both QC and RC regimes, where $\phi_{q} \sim 1 / q$ and $\omega_{q} \simeq c q$. According to Ref. [12], the one-magnon neutron scattering intensity in the insulator is indeed well described by Eq.(3) with $\gamma_{q} \ll \omega_{q}$.

It has been conjectured in Ref. [9] that the small doping as well as randomness related to it are not likely to affect the universal scaling behavior at high temperatures. Quite generally, one would expect a departure from $z=1$ behavior only when spin waves become overdamped by the electron-hole continuum [13]. Since this would require a substantial increase in spin wave damping, to $\gamma_{q}>\omega_{q}$, compared to its insulator value, $\gamma_{q} \ll \omega_{q}$, there may be an intermediate regime in which the spin waves are not yet absorbed by the electronhole continuum, even if the damping is increased compared to the insulator. To the extent this occurs, the system can remain in the $\mathrm{QC}$ regime with $z=1$ in a wide range of doping 
and temperatures due to the Lorentz invariant terms in the action.

In the insulator, the zero temperature energy gap $\Delta=\hbar c / \xi$ for the spin-1 excitations in a quantum disordered (QD) regime is, again, related to the Lorentz invariance at $T=0$ [7]. Hence, as long as the Lorentz invariant terms in the action are still important and the correlation length saturates, the low frequency $(\omega<\Delta)$ spectral weight could be suppressed even in a metal. We suggest that the spin gap phenomenon in $\mathrm{YBa}_{2} \mathrm{Cu}_{3} \mathrm{O}_{6.63}$ and $\mathrm{YBa}_{2} \mathrm{Cu}_{4} \mathrm{O}_{8}$, characterized by a sharp increase in $T_{1} T$ and decrease of the bulk susceptibility, is related to this suppression, and this phase corresponds to a $\mathrm{QD}, z=1$, regime.

At larger hole densities, the spin waves will be fully damped by the electron-hole continuum. In this regime, the self-consistent renormalization (SCR) approach developed by Moriya et al. [14] (see also Ref. [13]) and the phenomenological theory of Millis et al. [15], for $\mathrm{YBa}_{2} \mathrm{Cu}_{3} \mathrm{O}_{7}$ may be expected to apply. The dynamical exponent $z=2$, while $\eta=0$; in contrast with the previous case, the mean field exponent $z=2$ is not fixed by a symmetry, but rather follows from the scaling analysis of Ref. [14, 13]. One obtains $T_{1} T / T_{2 \mathrm{G}}^{2} \sim \bar{\omega} \xi^{2} \sim \xi^{2-z} \simeq$ const, while $T_{1} T / T_{2 \mathrm{G}} \sim \xi^{-1}$. At high temperatures, the energy scale $\bar{\omega} \sim T$, so that $\xi \sim \bar{\omega}^{-1 / z} \sim T^{-1 / z} \sim T^{-1 / 2}$, in which case $T_{1} T / T_{2 \mathrm{G}} \sim T^{1 / 2}$, and, separately, $1 / T_{1} \sim T \xi^{z} \simeq$ const, $1 / T_{2 \mathrm{G}} \sim \xi \sim T^{-1 / 2}$. We emphasize that $1 / T_{1} \simeq$ const at high temperatures is predicted for both $z=1$ and $z=2$ regimes, while predictions for $1 / T_{2 \mathrm{G}}$ are different. Finally, at still larger hole densities, the short range AF correlations between spins will tend to disappear; in this limit, $\xi \lesssim a$ is independent of temperature, and one recovers the Korringa law, $1 / T_{1} \sim T$, while $1 / T_{2 \mathrm{G}} \simeq$ const. This regime corresponds to a normal metal, in which any remaining antiferromagnetic correlations can be described by a temperature independent $F^{a}\left(\mathbf{p}, \mathbf{p}^{\prime}\right)$.

\section{3. $\mathrm{La}_{2-x} \mathrm{Sr}_{x} \mathrm{CuO}_{4}$}

We first consider $\mathrm{La}_{2-x} \mathrm{Sr}_{x} \mathrm{CuO}_{4}$. The insulator, $\mathrm{La}_{2} \mathrm{CuO}_{4}$, is well described by the 2D Heisenberg model with the nearest-neighbor exchange coupling $J \simeq 1500 K$, except near or below $T_{N} \sim 300 \mathrm{~K}$ induced by weak interplanar coupling [16]. A nearly temperature 
independent $1 / T_{1}$ is observed in the insulating $\mathrm{La}_{2} \mathrm{CuO}_{4}$ above $650 \mathrm{~K}$ [2], as expected in the QC, $z=1$, regime [9]. The absolute value of $1 / T_{1} \simeq 2700 \mathrm{sec}^{-1}$ at high temperatures [2] is in very good agreement with both $1 / N$ expansion [9] and finite cluster [11] calculations for the Heisenberg model. Further, the ratio $T_{1} T / T_{2 \mathrm{G}}$ measured in the insulator [2.:4] is nearly temperature independent in the broad range $450 \mathrm{~K}<T<900 \mathrm{~K}$ (Fig.2). This is again what one would expect in the $\mathrm{QC}, z=1$, regime; we note that this behavior holds even in the region below $650 \mathrm{~K}$, where $1 / T_{1}$ and $T / T_{2 \mathrm{G}}$ separately deviate from constant values, apparently because $T_{1} T / T_{2 \mathrm{G}}$ is insensitive to the magnitude of $\chi(\mathbf{q}, \omega)$.

A finite cluster calculation in the $S=1 / 2$ 2D Heisenberg model by E. Gagliano, S. Bacci, and one of the authors (A.S.) (Ref. [11] and this work), with no adjustable parameters used (hyperfine and exchange couplings were determined from other experiments, see Ref. [11]), indeed yields a nearly temperature independent $T_{1} T / T_{2 \mathrm{G}} \simeq 4.3 \cdot 10^{3} \mathrm{~K}^{-1}$ for $T>J / 2 \simeq 750 \mathrm{~K}$ (Fig.Q), in excellent agreement with the experimental result, $4.5 \cdot 10^{3} \mathrm{~K}$ [2]. The systematic error of the finite cluster calculation, arising from the periodic boundary conditions as well as spin diffusion contribution to $1 / T_{1}$, is estimated in Ref. [11] as $10-15 \%$.

An especially striking feature of the $1 / T_{1}$ data [2] is the nearly doping independent absolute value of $1 / T_{1}$ in the high temperature limit (Fig.2). This result shows that not only universal, but also nonuniversal scaling constants are not strongly renormalized in the doping range $x=0-0.15$, i.e. up to the optimal concentration for the superconductivity. Since it would be rather unrealistic to assume that exactly the same value can be obtained in different pictures of magnetism for low- and high-doped La-based materials, we suggest that the high temperature magnetic behavior over this entire doping range has the same physical origin as that found for the insulating state, which implies $z=1$. As the doping increases, Imai, Slichter, and collaborators [2] find that the range of temperatures where $T_{1} T$ is linear in temperature stretches towards lower temperatures, from $650 \mathrm{~K}$ for $x=0$ down to $125 \mathrm{~K}$ for $x=0.15$ (see Fig.2). This is the behavior expected if doping leads to a decrease in $\rho_{s}$, thus extending the QC region [9]. We thus conclude that $\mathrm{La}_{1.85} \mathrm{Sr}_{0.15} \mathrm{CuO}_{4}$ is in the quantum-critical, $z=1$, regime for $T \gtrsim 125 \mathrm{~K}$. 
As it is evident from Fig.2 (inset), for $T \lesssim 125 \mathrm{~K}, T_{1} T$ begins to depart from its linear in $T$ behavior, exhibiting an upturn for $T \approx 60-70 \mathrm{~K}$. We attribute this effect to the suppression of the low frequency spectral weight in the quantum disordered, $z=1$, regime [17]. At lower doping values, experiment shows that the low temperature phase is not a superconductor but rather a spin glass, in agreement with the scaling analysis [8], while for the lowest doping, the low temperature phase is the antiferromagnetic Neel state. 


\section{4. $\mathrm{YBa}_{2} \mathrm{Cu}_{3} \mathrm{O}_{6.63}$ and $\mathrm{YBa}_{2} \mathrm{Cu}_{4} \mathrm{O}_{8}$}

$\mathrm{YBa}_{2} \mathrm{Cu}_{3} \mathrm{O}_{6.63}$ and $\mathrm{YBa}_{2} \mathrm{Cu}_{4} \mathrm{O}_{8}$ have quite similar properties. The product $T_{1} T$ measured in $\mathrm{YBa}_{2} \mathrm{Cu}_{3} \mathrm{O}_{6.63}$ [18, [2], is linear in temperature for $160 \mathrm{~K} \lesssim T \lesssim 300 \mathrm{~K}$, while it exhibits similar behavior in $\mathrm{YBa}_{2} \mathrm{Cu}_{4} \mathrm{O}_{8}$ for $170 \mathrm{~K} \lesssim T \lesssim 800 \mathrm{~K}$. Since a linear $T_{1} T$ is predicted in both quantum-critical $(z=1)$ and overdamped $(z=2)$ regimes at high temperatures, to distinguish between these regimes, we turn to the $1 / T_{2 \mathrm{G}}$ data on $\mathrm{YBa}_{2} \mathrm{Cu}_{3} \mathrm{O}_{6.63}$ [3], and plot $T_{1} T / T_{2 \mathrm{G}}$ and $T_{1} T / T_{2 \mathrm{G}}$ as a function of temperature (Fig.3). In the range $200 \mathrm{~K}<T<300 \mathrm{~K}, T_{1} T / T_{2 \mathrm{G}}$ is nearly constant, while $T_{1} T / T_{2 \mathrm{G}}^{2}$ varies significantly, in agreement with the prediction for $z=1$. Were this material in the $z=2$ regime, $T_{1} T / T_{2 \mathrm{G}}$ would increase as the temperature increases, while $T_{1} T / T_{2 \mathrm{G}}^{2}$ would be constant. We thus conclude that above $200 \mathrm{~K}, \mathrm{YBa}_{2} \mathrm{Cu}_{3} \mathrm{O}_{6.63}$, and the closely related $\mathrm{YBa}_{2} \mathrm{Cu}_{4} \mathrm{O}_{8}$, are in the $\mathrm{QC}, z=1$, regime. The increase in damping in the doped case, which enhances $1 / T_{1}$ with respect to $1 / T_{2 \mathrm{G}}$, may explain the smaller (compared to the insulating $\mathrm{La}_{2} \mathrm{CuO}_{4}$, Fig.2) saturation value of $T_{1} T / T_{2 \mathrm{G}}$ in those compounds. Our scenario may seem to contradict the Raman studies in $\mathrm{YBa}_{2} \mathrm{Cu}_{3} \mathrm{O}_{6+x}$, because two-magnon Raman scattering is not observed for doping above $\mathrm{O}_{6.4}[19]$. However, this contradiction is illusory, since the decrease of intensity in the two-magnon Raman scattering is primarily due to the loss of the charge-transfer states rather than any change in the short range magnetic correlations [19,20].

For temperatures below $150 \mathrm{~K}, 1 / T_{1}$ sharply drops down as the temperature decreases, while $1 / T_{2 \mathrm{G}}$ [18,3] saturates. As was the case for $\mathrm{La}_{1.85} \mathrm{Sr}_{0.15} \mathrm{CuO}_{4}$, we argue this suppression of the low frequency spectral weight (spin gap) reflects a crossover to the quantum disordered, $z=1$, regime. Since in this regime the magnitude of the gap is inversely proportional to the correlation length, which is smaller in $\mathrm{YBa}_{2} \mathrm{Cu}_{3} \mathrm{O}_{6.63}$ and $\mathrm{YBa}_{2} \mathrm{Cu}_{4} \mathrm{O}_{8}$ than in $\mathrm{La}_{1.85} \mathrm{Sr}_{0.15} \mathrm{CuO}_{4}$, the onset temperature of the quantum disordered (spin gap) regime is larger and the crossover to it is more pronounced in the former two materials. Thus, unlike the scenario proposed by Millis and Monien [17], we argue that for all three materials the physical origin of the spin gap is the same. 


\section{5. $\mathrm{YBa}_{2} \mathrm{Cu}_{3} \mathrm{O}_{7}$}

For nearly stoichiometric $\mathrm{YBa}_{2} \mathrm{Cu}_{3} \mathrm{O}_{6.9}, T_{1} T / T_{2 \mathrm{G}}$ [1] increases as the temperature increases, while $T_{1} T / T_{2 \mathrm{G}}^{2}$ is nearly constant (Fig. 3), in agreement with the scaling prediction for the overdamped, $z=2$, regime [14]. The departure from the Korringa law $1 / T_{1} \sim T$ and large copper-to-oxygen ratio of the relaxation rates shows that the antiferromagnetic enhancement is still quite substantial. In the overdamped regime, the spin wave branch is either destroyed, or due to the small correlation length has appreciable spectral weight only for energies much larger than the maximal temperature at which this compound is chemically stable. Therefore, for experimentally accessible temperatures no departure from the the overdamped regime is observed.

\section{Conclusion}

We have shown that the nuclear relaxation data in a broad range of doping levels, which includes both metallic and insulating materials, possesses universal features characteristic of the quantum critical regime of a clean antiferromagnetic insulator with the dynamical exponent $z=1$. This universality suggests that well beyond the metal-insulator transition, the spin excitation spectrum of the insulator is not yet destroyed by the electron-hole background, in which case one expects a two-component dynamics for a broad range of doping levels. While a two-component dynamics may arise in a one-component as well as a twocomponent microscopic model, we call attention to an explicit example which leads to this kind of dynamics directly, namely, a model of spins and fermions with both spin-spin $(J)$

and weak spin-fermion $(\widetilde{J})$ exchange interaction [21]. The robustness of the spin waves may be related to either their weak coupling to quasiparticles, or to quasiparticle Fermi surfaces which are not spanned by the antiferromagnetic ordering vector $\mathbf{Q}$.

On the basis of the above analysis, we suggest the unified magnetic phase diagram for the cuprate superconductors shown in Fig.1; the proposed boundary between the QC and QD regimes is determined from the nuclear relaxation data shown on Fig.2. We propose 
that as the hole doping increases, the transition from the insulating to the overdoped regime occurs in two stages. First, the system becomes metallic; the damping of spin waves increases somewhat compared to its value in the insulator, but since the spin waves are not destroyed by the electron-hole background, the dynamical exponent is $z=1$ and the quantum critical regime persists over a wide range of temperatures and doping levels. Then, at substantially higher doping, the dynamical exponent crosses over to $z=2$. We further argue that the spin gap phenomenon observed in the underdoped materials reflects the same physics as the formation of the gap for spin excitations in the the quantum disordered, $z=1$, phase of an insulator. This scenario suggests that in compounds where the spin gap is observed, the temperature dependent bulk susceptibility should exhibit a downturn near the crossover from the QC to QD regime, while $T_{1} T / T_{2 \mathrm{G}}$ should be temperature independent at higher temperatures. We show, in a subsequent communication [22], that our scenario leads in a natural way to a unified description of the results of nuclear relaxation, magnetic susceptibility, and neutron scattering experiments.

\section{Acknowledgements}

We are grateful to M. Takigawa, T. Imai, and C.P. Slichter for communicating their experimental data to us in advance of publication. We would like to thank V. Barzykin, G. Blumberg, A.V. Chubukov, S.L. Cooper, E. Dagotto, D. Frenkel, L.P. Gor'kov, M.V. Klein, A.J. Millis, and D. Thelen for valuable discussions, and T. Imai and C.P. Slichter for numerous stimulating conversations on nuclear resonance phenomena. This work has been supported by the NSF Grant DMR89-20538 through the Materials Research Laboratory. 


\section{REFERENCES}

[1] T. Imai, C.P. Slichter, A.P. Paulikas, and B. Veal, Phys. Rev. B 47, 9158 (1993).

[2] T. Imai, C.P. Slichter, K. Yoshimura, and K. Kosuge, Phys. Rev. Lett. 70, 1002 (1993).

[3] M. Takigawa, private communication.

[4] T. Imai, C.P. Slichter, K. Yoshimura, M. Katoh, and K. Kosuge, to be published.

[5] C.H. Pennington and C.P. Slichter, Phys. Rev. Lett. 66, 381 (1991); see also D. Thelen and D. Pines, preprint (1993).

[6] R.R.P. Singh, Phys. Rev. B 39, 9760 (1989).

[7] S. Chakravarty, B.I. Halperin, and D.R. Nelson, Phys. Rev. B 39, 2344 (1989).

[8] S. Sachdev and J. Ye, Phys. Rev. Lett. 69, 2411 (1992).

[9] A.V. Chubukov and S. Sachdev, preprint No. 9301027; A.V. Chubukov, S. Sachdev, and J. Ye, preprint No. 9304046 in cond-mat@babbage.sissa.it.

[10] M. Makivić and M. Jarrell, Phys. Rev. Lett. 68, 1770 (1992).

[11] A. Sokol, E. Gagliano, and S. Bacci, Phys. Rev. B, June 1, 1993 (in press; see No. 9302013 in cond-mat@babbage.sissa.it); and to be published.

[12] S.M. Hayden, G. Aeppli, R. Osborn, A.D. Taylor, T.G. Perring, S.-W. Cheong, and Z. Fisk, Phys. Rev. Lett. 67, 3622 (1991).

[13] A.J. Millis, preprint (1993).

[14] T. Moriya, Y. Takahashi, and K. Ueda, Phys. Rev. Lett. 59, 2905 (1990), and references therein.

[15] A.J. Millis, H. Monien, and D. Pines, Phys. Rev. B 42, 167 (1990).

[16] S. Chakravarty, in Proceedings of High Temperature Superconductivity, edited by K.S. 
Bedell et al. (Addison-Wesley, CA, 1990); E. Manousakis, Rev. Mod. Phys. 63, 1 (1991).

[17] A.J. Millis and H. Monien [Phys. Rev. Lett. 70, 2810 (1993)] have suggested that temperature dependent bulk susceptibility in $\mathrm{YBa}_{2} \mathrm{Cu}_{3} \mathrm{O}_{6.63}$ is due to the gap for spin excitations induced by the interplanar coupling, while in $\mathrm{La}_{1.85} \mathrm{Sr}_{0.15} \mathrm{CuO}_{4}$ it is caused by the SDW fluctuations, in contrast with our common mechanism for both Y- and La-based materials. The spin gap regime has been discussed in a number of publications; some of them are listed as Ref.7 of the above paper.

[18] M. Takigawa, A.P. Reyes, P.C. Hammel, J.D. Thompson, R.H. Heffner, Z. Fisk, and K.C. Ott, Phys. Rev. B 43, 247 (1991).

[19] S.L. Cooper, D. Reznik, A. Kotz, M.A. Karlow, R. Liu, M.V. Klein, W.C. Lee, J. Giapintzakis, and D.M. Ginsberg, Phys. Rev. B, 47, 8233 (1993).

[20] We thank M.V. Klein for pointing this out to us.

[21] J.R. Schrieffer, L. Lilly, and N.E. Bonesteel, APS 1993 March Meeting abstract G23-1, and private communication; see also L.P. Gor'kov and A. Sokol, Physica C 159, 329 (1989).

[22] V. Barzykin, D. Pines, A. Sokol, and D. Thelen, to be published.

[23] Y. Kitaoka, S. Ohsugi, K. Ishida, and K. Asayama, Physica C 189, 189 (1990).

[24] T. Machi, I. Tomeno, T. Miyatake, N. Koshizuka, S. Tanaka, T. Imai, and H. Yasuoka, Physica C 173, 32 (1991). 


\section{FIGURES}

FIG. 1. The proposed magnetic phase diagram for the cuprate superconductors above 100K.

FIG. 2. Experimental data on $T_{1} T$ and $T_{1} T / T_{2 \mathrm{G}}: \otimes \mathrm{La}_{2} \mathrm{CuO}_{4}$ [2,

$\nabla \mathrm{La}_{1.85} \mathrm{Sr}_{0.15} \mathrm{CuO}_{4}$ [2]; $\Delta \mathrm{La}_{1.85} \mathrm{Sr}_{0.15} \mathrm{CuO}_{4}$ [23]; - $\mathrm{YBa}_{2} \mathrm{Cu}_{4} \mathrm{O}_{8}$ [24]; $\circ \mathrm{YBa}_{2} \mathrm{Cu}_{3} \mathrm{O}_{6.63}$ [18,3]; $\mathrm{YBa}_{2} \mathrm{Cu}_{3} \mathrm{O}_{6.9}$ [1]. Also shown $(\diamond)$ are the results of numerical calculation of $1 / T_{1}$ [1]] and $1 / T_{2 \mathrm{G}}$ ( 11] and this work) for the insulator. The arrows indicate our proposed values for the crossover temperature from the QC to QD regimes; the inset makes clear our choice for $\mathrm{La}_{1.85} \mathrm{Sr}_{0.15} \mathrm{CuO}_{4}$.

FIG. 3. Experimental data on $T_{1} T / T_{2 \mathrm{G}}$ and $T_{1} T / T_{2 \mathrm{G}}^{2}: \circ \mathrm{YBa}_{2} \mathrm{Cu}_{3} \mathrm{O}_{6.63}$ [18,3];

- $\mathrm{YBa}_{2} \mathrm{Cu}_{3} \mathrm{O}_{6.9}$ [䝅]. 